\title{
Performance Limits of Fluid Antenna Systems
}

\author{
Kai-Kit Wong, Fellow, IEEE, Arman Shojaeifard, Kin-Fai Tong, Senior Member, IEEE, and Yangyang Zhang
}

\begin{abstract}
Fluid antenna represents a concept where a positionflexible antenna can switch its location freely within a given space. Recently, it has been demonstrated that even with a tiny space, a single-antenna fluid antenna system (FAS) can outperform an $L$-antenna maximum ratio combining (MRC) system in terms of outage probability if the number of locations (or ports) the fluid antenna can be switched to, is large enough. This letter aims to study if extraordinary capacity can also be achieved by FAS with a small space. We do this by deriving the ergodic capacity, and a capacity lower bound. This letter also derives the level crossing rate (LCR) and average fade duration (AFD) for the FAS.
\end{abstract}

Index Terms-Capacity, Fluid antennas, MIMO.

\section{INTRODUCTION}

A main challenge in mobile phone design is the increasing limited physical dimensions. It is common practice that multiple antennas are only deployed, if they are sufficiently apart. The rule of thumb is that antennas should have a separation of at least $\frac{\lambda}{2}$ where $\lambda$ is the wavelength. However, this approach may need to be changed because the intuition that a tiny space does not have rich diversity is far from accurate.

In [1], a novel fluid antenna system (FAS) was investigated, where the antenna can be switched to one of $N$ fixed locations in a linear space. The work was motivated by the emergence of mechanically flexible antennas, some of which are based on liquid metal antennas, e.g., [2]-[5] or ionized solutions [6]-[8], while others utilize pixel antennas [9]. The beauty of 'fluid' antennas is that an antenna is no longer fixed at a location but can be switched to a more favourable location if needed.

The rationale of FAS resembles traditional antenna selection with one RF chain. However, for traditional antenna selection systems, multiple antennas are deployed at fixed locations and the antenna with the strongest signal is selected. By contrast, in FAS, there is only one antenna whose position (referred to as "port") is flexible within a predefined space. FAS selects the best port for the strongest reception in the same way as choosing the best antenna in a multiple antenna system. Note that FAS may be realized by different ways. One way is to use conductive fluids or liquid metals which can be mobilized in a tube-like structure using a software-controlled microfluidic system, e.g., [2]-[8]. Alternatively, a "fluid" antenna can also be realized by an array of pixel-like electronic switches [9]. By turning on or off the pixels, an antenna can be made to appear or disappear instantly in a given space.

The work is supported by EPSRC under grant EP/M016005/1.

$\mathrm{K}$. Wong and K. Tong are with the Department of Electronic and Electrical Engineering, University College London, London WC1E 7JE, United Kingdom. E-mail: \{kai-kit.wong, k.tong@ucl.ac.uk\}.

A. Shojaeifard is with BT Labs, Ipswich, United Kingdom. E-mail: arman.shojaeifard@bt.com.

Y. Zhang is with Kuang-Chi Institute of Advanced Technology, China. Email: yangyang.zhang@kuang-chi.org.
A key finding in [1] is that though space matters, a singleantenna FAS with a tiny space can achieve any arbitrarily small outage probability and surpass a multi-antenna maximum ratio combining (MRC) system, if $N$ is large enough. The aim of this letter is to examine if the promising outage performance of FAS can be translated into other benefits. In particular, this letter derives the level crossing rate (LCR), the average fade duration (AFD), and the ergodic capacity for the $N$-port FAS. One major contribution is a closed-form capacity lower bound for the FAS. Numerical results confirm the huge capacity gain unfolded from the diversity hidden in a small space of FAS.

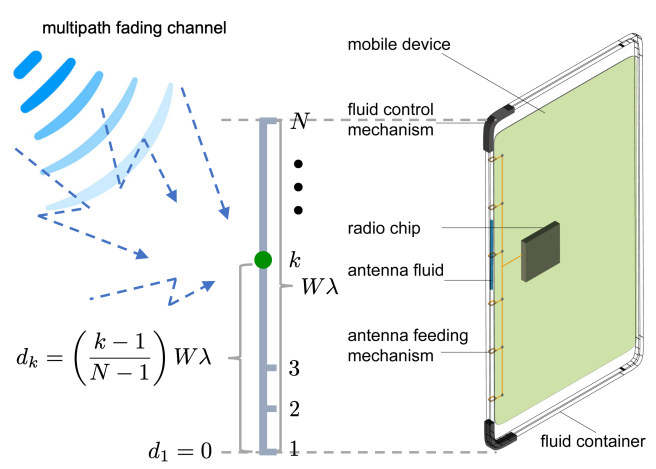

Fig. 1. The concept of FAS. In practice, the relocation of the antenna can be realized by mechanically moving liquid conductors using a microfluidic system, or electronically turning on or off radiating pixels.

\section{FAS SYSTEM MODEL}

We consider a FAS with linear space of $W \lambda$ where $\lambda$ is the wavelength, as shown in Figure 1. It is a single-antenna system where the antenna is mechanically flexible, using technologies such as microfluidic systems [5]-[7] or on-off pixels [9], and can be switched to one of the $N$ preset locations along the space. ${ }^{1}$ We refer to the location as port, and the ports are evenly distributed over the space of $W \lambda$, sharing one RF chain.

The received signal at the $k$-th port can be written as

$$
y_{k}=g_{k} x+\eta_{k},
$$

in which $x$ is the transmitted symbol, $\eta_{k}$ is the additive white Gaussian noise (AWGN) with zero mean and variance of $\sigma_{\eta}^{2}$, $g_{k}$ is the complex channel envelope, and $r_{k}=\left|g_{k}\right|$ is Rayleigh distributed, with the probability density function (pdf)

$$
p_{r_{k}}(r)=\frac{2 r}{\sigma^{2}} e^{-\frac{r^{2}}{\sigma^{2}}}, \text { for } r_{k} \geq 0,
$$

with $\mathrm{E}\left[r_{k}^{2}\right]=\sigma^{2}$. Also, we define the average signal-to-noise ratio (SNR) as $\Gamma \triangleq \sigma^{2} \frac{\mathrm{E}\left[|x|^{2}\right]}{\sigma_{\eta}^{2}}=\sigma^{2} \Theta$.

\footnotetext{
${ }^{1}$ In this letter, the switching delay is assumed negligible, which is particularly reasonable in the case of pixel-based fluid antenna technologies.
} 
We parameterize the channels for the $N$-port FAS by

$$
\left\{\begin{aligned}
& g_{1}=\sigma x_{0}+j \sigma y_{0} \\
& g_{k}=\sigma\left(\sqrt{1-\mu_{k}^{2}} x_{2}+\mu_{k} x_{0}\right) \\
&+j \sigma\left(\sqrt{1-\mu_{k}^{2}} y_{2}+\mu_{k} y_{0}\right), \text { for } 1<k \leq N,
\end{aligned}\right.
$$

where $x_{0}, x_{1}, \ldots, x_{N}, y_{0}, y_{1}, \ldots, y_{N}$ are all independent Gaussian random variables with zero mean and variance of $\frac{1}{2}$, and $\left\{\mu_{k}\right\}$ are the parameters that can be chosen freely to control the correlation between the channels $\left\{g_{k}\right\}$. Assuming 2-D isotropic scattering and isotropic receiver ports on the FAS, the autocorrelation parameters are given by [1], [10]

$$
\mu_{k}=J_{0}\left(\frac{2 \pi(k-1)}{N-1} W\right), \text { for } k=2, \ldots, N,
$$

where $J_{0}(\cdot)$ is the zero-order Bessel function of the first kind.

In this letter, we assume that the FAS can always select the best port with the strongest signal for communication, i.e.,

$$
r_{\mathrm{FAS}}=\max \left\{r_{1}, r_{2}, \ldots, r_{N}\right\} .
$$

\section{LCR AND AFD}

To analyze LCR and AFD, we need the time-derivatives of the signal envelopes, $\left\{\dot{r}_{k}\right\}_{\forall k}$. According to [11], for isotropic scattering, $\dot{r}_{k}$ is Gaussian distributed with zero mean and variance $\hat{\sigma}^{2}=2 \pi^{2} \sigma^{2} f_{m}^{2}$ where $f_{m}=v / \lambda$ is the maximum Doppler shift for speed $v$.

Theorem 1: The LCR for FAS is given by

$$
N_{r_{\mathrm{FAS}}}(r)=\frac{2 \sqrt{\pi} v}{\sigma \lambda} r e^{-\frac{r^{2}}{\sigma^{2}}}
$$

Proof: From [12, (14)], we have

$$
\begin{aligned}
N_{r_{\mathrm{FAS}}}(r) & =\int_{0}^{\infty} \dot{r} p_{r_{\mathrm{FAS}} \dot{r}_{\mathrm{FAS}}}(r, \dot{r}) d \dot{r} \\
& =\sum_{j=1}^{N} N_{r_{\mathrm{FAS}} \mid r_{j}}\left(r \mid r_{j}\right) \operatorname{Prob}\left(r_{j}=r_{\mathrm{FAS}}\right)=\frac{\hat{\sigma} p_{r}(r)}{\sqrt{2 \pi}}
\end{aligned}
$$

where $p_{r}(r)$ is given by (2). We then obtain (6) after using (2) and the fact that $\hat{\sigma}^{2}=2 \pi^{2} \sigma^{2} f_{m}^{2}=2 \pi^{2} \sigma^{2} v^{2} / \lambda^{2}$.

Theorem 2: The AFD for an $N$-port FAS, for a level $r$, is given by

$$
\tau_{r_{\mathrm{FAS}}}(r)=\frac{\operatorname{Prob}\left(r_{\mathrm{FAS}} \leq r\right)}{N_{r_{\mathrm{FAS}}}(r)},
$$

where the outage probability is given by [1, Theorem 3] and $N_{r_{\mathrm{FAS}}}(r)$ is obtained by (6).

Proof: The result comes from the definition of AFD.

\section{ERgodic CAPACITY}

Lemma 1: The ergodic capacity can be obtained by

$$
\mathrm{E}[\ln (1+\gamma)]=\int_{0}^{\infty}\left(\frac{1}{1+y}\right) \operatorname{Prob}\left(r>\sqrt{\frac{y}{\Theta}}\right) d y,
$$

(in nats/channel-use),

where $\gamma=r^{2} \Theta$ is the instantaneous SNR.
Proof: Using $\mathrm{E}[Y]=\int_{0}^{\infty} \operatorname{Prob}(Y>x) d x$, we get

$$
\begin{aligned}
\mathrm{E}[\ln (1+\gamma)] & =\mathrm{E}\left[\ln \left(1+r^{2} \Theta\right)\right] \\
& =\int_{0}^{\infty} \operatorname{Prob}\left(\ln \left(1+r^{2} \Theta\right)>x\right) d x \\
& =\int_{0}^{\infty} \operatorname{Prob}\left(r^{2} \Theta>e^{x}-1\right) d x .
\end{aligned}
$$

Then by changing the variable $y=e^{x}-1$, we get (9).

The following theorem establishes the exact ergodic capacity expression for the FAS in an analytical form.

Theorem 3: The ergodic capacity of the FAS is given by

$$
\begin{aligned}
& C_{\mathrm{FAS}}=\int_{0}^{\infty}\left(\frac{1}{1+y}\right)\left\{1-\int_{0}^{\frac{y}{\Gamma}} e^{-t} \times\right. \\
& \left.\prod_{k=2}^{N}\left[1-Q_{1}\left(\sqrt{\frac{2 \mu_{k}^{2}}{1-\mu_{k}^{2}}} \sqrt{t}, \sqrt{\frac{2}{1-\mu_{k}^{2}}} \sqrt{\frac{y}{\Gamma}}\right)\right] d t\right\} d y,
\end{aligned}
$$

(in nats/channel-use),

where $Q_{1}(\cdot, \cdot)$ denotes the first-order Marcum $Q$-function.

Proof: Using Lemma 1 and noting that

$$
\operatorname{Prob}\left(r>\sqrt{\frac{y}{\Theta}}\right)=1-\operatorname{Prob}\left(r_{\mathrm{FAS}} \leq \sqrt{\frac{y}{\Theta}}\right),
$$

we obtain (11) after substituting $\Gamma=\sigma^{2} \Theta$.

Theorem 3 provides the exact result for the ergodic capacity but it is difficult to gain any insight. We now try to establish a lower bound in a closed form so that the capacity benefit for the spatially correlated ports can be quantified.

Lemma 2: For $0<\alpha<\beta$ and large $\beta$, we have the following lower bound for $Q_{1}(\alpha, \beta)$ :

$$
Q_{1}(\alpha, \beta) \gtrsim \varrho \sqrt{\frac{\beta}{\alpha}} e^{-\frac{\kappa}{2}(\beta-\alpha)^{2}},
$$

where $\kappa$ is any positive constant greater than one, and $\varrho \triangleq$ $\frac{e^{\overline{\pi(\kappa-1)+2}}}{2 \kappa} \sqrt{\frac{(\kappa-1)(\pi(\kappa-1)+2)}{\pi}}$. Note that $0<\varrho<0.5$.

Proof: See [1, Lemma 6].

Theorem 4: Defining the service probability of an $N$-port FAS, for a level $y$, as

$$
q_{N}(y) \triangleq \operatorname{Prob}\left(r_{\mathrm{FAS}}>\sqrt{\frac{y}{\Theta}} \mid N\right),
$$

it can be lower-bounded by

$$
q_{N}(y) \geq \sum_{\substack{\ell=1 \\ 1 \leq k_{1}, \ldots, k_{\ell} \leq N \\ k_{\ell_{1}} \neq k_{\ell_{2}}}}^{N}(-1)^{\ell+1} \varepsilon_{k_{1}} \varepsilon_{k_{2}} \cdots \varepsilon_{k_{\ell}}
$$

where

$$
\varepsilon_{k} \triangleq\left\{\begin{array}{c}
\frac{\varrho}{\sqrt{\left|\mu_{k}\right|}} e^{-\frac{\kappa}{1-\mu_{k}^{2}}\left(\frac{y}{\Gamma}\right)}, \text { if } k>1 \text { and }\left|\mu_{k}\right|>\varrho^{2} \\
e^{-\frac{\kappa}{1-\mu_{k}^{2}}\left(\frac{y}{\Gamma}\right)}, \text { if } k>1 \text { and }\left|\mu_{k}\right| \leq \varrho^{2} \\
e^{-\frac{y}{\Gamma}}, \text { if } k=1,
\end{array}\right.
$$

in which $\kappa>1$ and $0<\varrho<0.5$ are defined in Lemma 2 . 
Proof: First of all, define the outage probability so that

$$
p_{N}(y)=\operatorname{Prob}\left(r_{\mathrm{FAS}} \leq \sqrt{\frac{y}{\Theta}} \mid N\right)=1-q_{N}(y) .
$$

A lower bound of $q_{N}(y)$ can be obtained by developing an upper bound for $p_{N}(y)$. Denoting $\alpha_{k}=\sqrt{\frac{2 \mu_{k}^{2}}{1-\mu_{k}^{2}}}$ and $\beta_{k}=$ $\sqrt{\frac{2}{1-\mu_{k}^{2}}} \sqrt{\frac{1}{\Gamma}}$, we can find that

$$
\begin{array}{r}
p_{N}(y)=p_{N-1}(y)-\int_{0}^{\frac{y}{\Gamma}} Q_{1}\left(\alpha_{N} \sqrt{t}, \beta_{N} \sqrt{y}\right) \times \\
e^{-t} \prod_{k=2}^{N-1}\left[1-Q_{1}\left(\alpha_{k} \sqrt{t}, \beta_{k} \sqrt{y}\right)\right] d t .
\end{array}
$$

As $\alpha_{N} \sqrt{t} \leq \beta_{N} \sqrt{y}$, we use Lemma 2 to get

$$
Q_{1}\left(\alpha_{N} \sqrt{t}, \beta_{N} \sqrt{y}\right) \gtrsim \frac{\varrho}{\sqrt{\left|\mu_{N}\right|}} \frac{\left(\frac{y}{\Gamma}\right)^{0.25}}{t^{0.25}} e^{-\frac{\kappa}{1-\mu_{N}^{2}}\left(\sqrt{\frac{y}{\Gamma}}-\mu_{N} \sqrt{t}\right)^{2}} \text {. }
$$

As $0 \leq t \leq \frac{y}{\Gamma}$, we can further have the following lower bound

$$
Q_{1}\left(\alpha_{N} \sqrt{t}, \beta_{N} \sqrt{y}\right) \gtrsim \frac{\varrho}{\sqrt{\left|\mu_{N}\right|}} e^{-\frac{\kappa}{1-\mu_{N}^{2}}\left(\frac{y}{\Gamma}\right)} \equiv \varepsilon_{N} .
$$

Now, using this result inside the integration of (18), we have

$$
\begin{array}{r}
p_{N}(y) \leq\left(1-\varepsilon_{N}\right) p_{N-1}(y) \leq\left(1-\varepsilon_{N}\right)\left(1-\varepsilon_{N-1}\right) p_{N-2}(y) \\
\cdots \leq p_{1}(y) \prod_{k=2}^{N}\left(1-\varepsilon_{k}\right) . \quad(21)
\end{array}
$$

As a result, we can obtain a lower bound for $q_{N}(y)$ by

$$
q_{N}(y) \geq 1-p_{1}(y) \prod_{k=2}^{N}\left(1-\varepsilon_{k}\right) .
$$

Note that $p_{1}(y)=1-e^{-\frac{y}{\Gamma}}=1-\varepsilon_{1}$. If we then expand the product in (22), then we have

$$
\prod_{k=1}^{N}\left(1-\varepsilon_{k}\right)=1+\sum_{\substack{\ell=1 \\ 1 \leq k_{1}, \ldots, k_{\ell} \leq N \\ k_{\ell_{1}} \neq k_{\ell_{2}}}}^{N}(-1)^{\ell} \varepsilon_{k_{1}} \varepsilon_{k_{2}} \cdots \varepsilon_{k_{\ell}} .
$$

Using the above result on (22) gives the desired result. Note that (21) is valid only if $\frac{\varrho}{\sqrt{\left|\mu_{k}\right|}} e^{-\frac{k}{1-\mu_{k}^{2}}\left(\frac{y}{\Gamma}\right)} \leq 1$. Therefore, the definition (16) is introduced to guarantee that.

Theorem 5: The ergodic capacity for an $N$-port FAS in (11) is lower bounded by

$$
\begin{array}{r}
C_{\mathrm{FAS}} \geq C_{\mathrm{LB}}=\sum_{\substack{\ell=1 \\
1 \leq k_{1}, \ldots, k_{\ell} \leq N \\
k_{\ell_{1}} \neq k_{\ell_{2}}}}^{N}(-1)^{\ell+1} a_{k_{1}} \cdots a_{k_{\ell}} \times \\
e^{\sum_{j=1}^{\ell} b_{k_{j}}} \Gamma_{\text {inc }}\left(0, \sum_{j=1}^{\ell} b_{k_{j}}\right),
\end{array}
$$

where $\Gamma_{\text {inc }}(x, y)$ is the upper incomplete Gamma function,

$$
a_{k} \triangleq\left\{\begin{array}{r}
1 \text { if } k=1 \text { or }\left|\mu_{k}\right| \leq \varrho^{2}, \\
\frac{\varrho}{\sqrt{\left|\mu_{k}\right|}} \text { if } k \neq 1 \text { and }\left|\mu_{k}\right|>\varrho^{2},
\end{array}\right.
$$

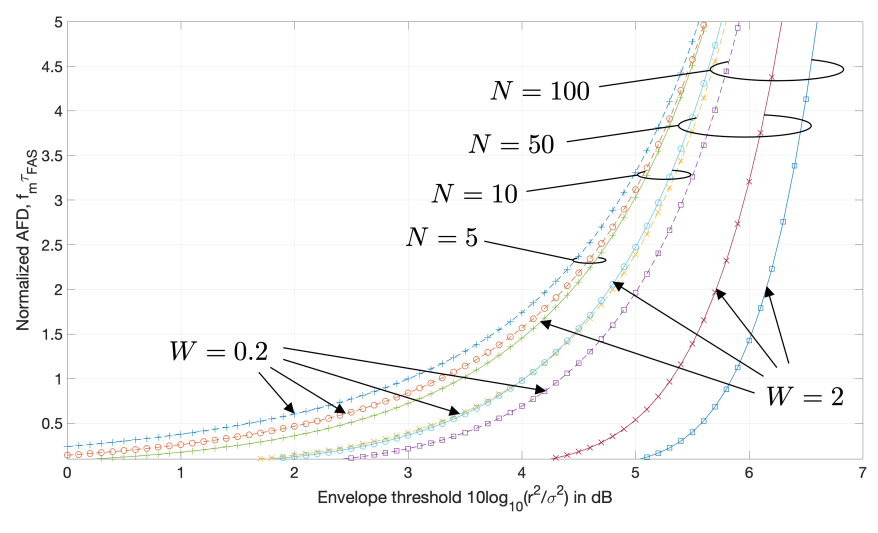

Fig. 2. The normalized AFD results against the level for various $W$ and $N$.

and

$$
b_{k} \triangleq\left\{\begin{array}{r}
\frac{1}{\Gamma} \text { if } k=1, \\
\frac{\kappa}{1-\mu_{k}^{2}}\left(\frac{1}{\Gamma}\right) \text { if } k \neq 1 .
\end{array}\right.
$$

Proof: From Lemma 1, we have

$$
C_{\mathrm{FAS}}=\int_{0}^{\infty} \frac{q_{N}(y)}{1+y} d y
$$

Using the lower bound for $q_{N}(y)$ in Theorem 4, we get

$$
\begin{aligned}
C_{\mathrm{FAS}} \geq & \sum_{\substack{\ell=1 \\
1 \leq k_{1}, \ldots, k_{\ell} \leq N \\
k_{\ell_{1}} \neq k_{\ell_{2}}}}^{N}(-1)^{\ell+1} \int_{0}^{\infty} \frac{\varepsilon_{k_{1}} \varepsilon_{k_{2}} \cdots \varepsilon_{k_{\ell}}}{1+y} d y \\
& =\sum_{\substack{\ell=1 \\
1 \leq k_{1}, \ldots, k_{\ell} \leq N \\
k_{\ell_{1}} \neq k_{\ell_{2}}}}^{N}(-1)^{\ell+1} a_{k_{1}} \cdots a_{k_{\ell}} \int_{0}^{\infty} \frac{e^{-\left(b_{k_{1}}+\cdots+b_{k_{\ell}}\right) y}}{1+y} d y .
\end{aligned}
$$

Using the fact that $\int_{0}^{\infty} \frac{e^{-c y}}{1+y} d y=e^{c} \Gamma_{\text {inc }}(0, c)$ gives the final result (24), which completes the proof.

\section{Numerical Results}

In this section, we provide the numerical results for the AFD and ergodic capacity under different settings. Figure 2 shows the AFD results against the signal envelope level for the FAS with different $N$ and size $W$. As expected, if the level is large, AFD eventually grows rapidly without bound. What is crucial is when this starts to happen. The results reveal that $N$ helps increase the signal level with practically zero AFD. Also, the size $W$ has a positive impact on the AFD. If $W$ is larger, more diversity potentially exists and AFD can be reduced.

Results in Figure 3 are provided for the ergodic capacity of the FAS. Figure 3(a) illustrates how the capacity of the FAS scales with $N$. As we can observe, capacity continues to increase with $N$ even when $W$ is very small. Also, $W=0.5$ appears to have the biggest jump in performance, as further increase in $W$ only contributes little. This may be explained by knowing the fact that a linear space for FAS has its spatial autocorrelation function following the Bessel function (4). It is well understood that the Bessel function has the biggest 
drop in the absolute value of the spatial autocorrelation when the distance is at $\frac{\lambda}{2}$. As such, we interpret that if $W=0.5$, then the FAS obtains the most benefit from the reduction in spatial correlation for capacity gain. In other words, further increase in the size will result in a diminishing return. This is further confirmed by the results in Figure 3(b), which show that capacity plateaus when $W$ reaches 1 . This reveals that $N$ is a more important factor than $W$ in the FAS. Remarkably, results also demonstrate that the capacity of a single-antenna FAS can obtain that of a multi-antenna MRC system with independent fading. In particular, $N=10$ will be enough for FAS with $W \geq 0.5$ to approach a 3-antenna MRC system. More capacity is also possible if $N$ continues to increase.

Lastly, results in Figure 3(c) evaluate the lower bound in Theorem 5 against the exact capacity for different values of SNR. The results indicate that although the lower bound is not particularly tight, it accurately picks up how the capacity grows with the SNR. Also, the lower bound scales very well with $N$. As we can see, the gap between the bound and the exact result remains similar if $N$ increases.

\section{CONCLUSION}

Following the emergence of mechanically flexible antennas, this letter studied if the diversity of FAS with a small space can be translated into substantial capacity gain. To this end, we first derived the exact ergodic capacity in an analytical form, and then established a capacity lower bound that was able to reveal how the capacity scales with the system parameters. We concluded that a single-antenna FAS, with a small space, can achieve the capacity of a multi-antenna MRC system.

\section{REFERENCES}

[1] K. K. Wong, A. Shojaeifard, K. F. Tong, and Y. Zhang, "Fluid antenna systems," [Online] arXiv:2005.11561 [cs.IT].

[2] K. N. Paracha, A. D. Butt, A. S. Alghamdi, S. A. Babale, and P. J. Soh, "Liquid metal antennas: Materials, fabrication and applications," Sensors 2020, 20, 177.

[3] G. J. Hayes, J.-H. So, A. Qusba, M. D. Dickey, and G. Lazzi, "Flexible liquid metal alloy (EGaIn) microstrip patch antenna," IEEE Trans. Antennas Propag., vol. 60, no. 5, pp. 2151-2156, May 2012.

[4] A. M. Morishita, C. Kitamura, A. T. Ohta, and W. A. Shiroma, "A liquidmetal monopole array with tunable frequency, gain, and beam steering," IEEE Antennas Wireless Propag. Lett., vol. 12, pp. 1388-1391, 2013.

[5] A. Dey, R. Guldiken, and G. Mumcu, "Microfluidically reconfigured wideband frequency-tunable liquid-metal monopole antenna," IEEE Trans. Antennas Propag., vol. 64, no. 6, pp. 2572-2576, Jun. 2016.

[6] C. Borda-Fortuny, K. F. Tong, A. Al-Armaghany, and K. K. Wong, "A low-cost fluid switch for frequency-reconfigurable Vivaldi antenna," IEEE Antennas Wireless Prop. Lett., vol. 16, pp. 3151-3154, Nov. 2017.

[7] C. Borda-Fortuny, K. F. Tong, and K. Chetty, "Low-cost mechanism to reconfigure the operating frequency band of a Vivaldi antenna for cognitive radio and spectrum monitoring applications," IET Microwaves, Antennas \& Propag., vol. 12, no. 5, pp. 779-782, 2018.

[8] A. Singh, I. Goode, and C. E. Saavedra, "A multistate frequency reconfigurable monopole antenna using fluidic channels," IEEE Antennas Wireless Propag. Lett., vol. 18, no. 5, pp. 856-860, May 2019.

[9] S. Song, and R. D. Murch, "An efficient approach for optimizing frequency reconfigurable pixel antennas using genetic algorithms," IEEE Trans. Antennas Propag., vol. 62, no. 2, pp. 609-620, Feb. 2014.

[10] G. L. Stüber, Principles of Mobile Communication, Second Edition, Kluwer Academic Publishers, 2002.

[11] W. Jakes, Microwave Mobile Communications. New York: Wiley, 1974.

[12] C. D. Iskander, and P. T. Mathiopoulos, "Analytical level crossing rates and average fade durations for diversity techniques in Nakagami fading channels," IEEE Trans. Commun., vol. 50, no. 8, pp. 1301-1309, Aug. 2002.

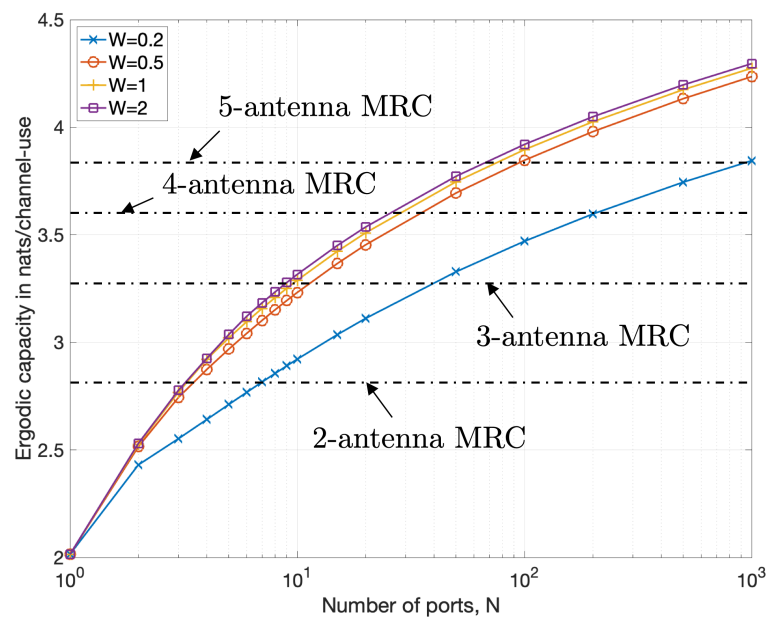

(a) Ergodic capacity versus number of ports when $\Gamma=10 \mathrm{~dB}$

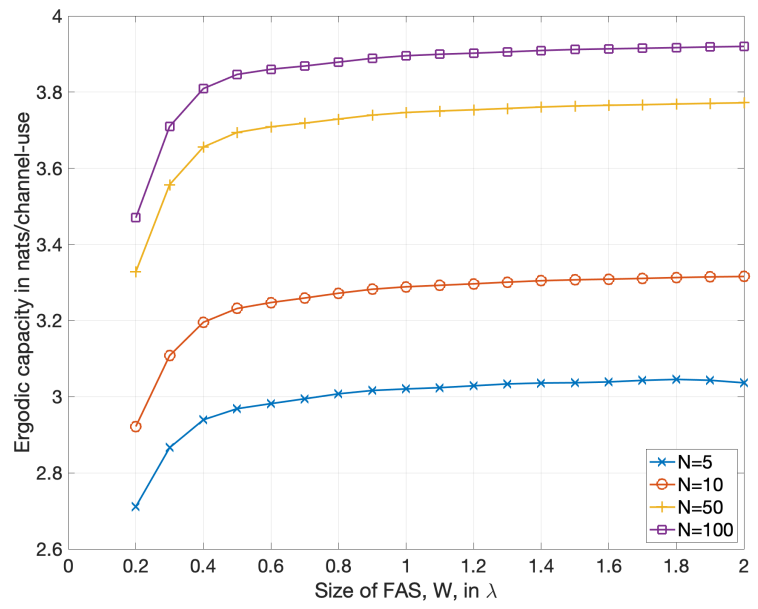

(b) Ergodic capacity versus size when $\Gamma=10 \mathrm{~dB}$

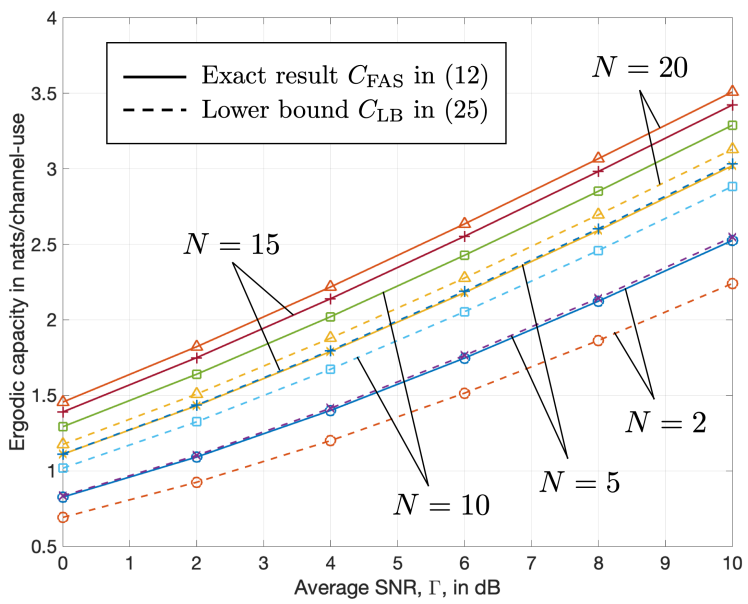

(c) Ergodic capacity versus the lower bound with $W=1$

Fig. 3. Ergodic capacity results for the FAS. 